\title{
Frontières
}

Hentsch, Thierry, Raconter et mourir. Aux sources narratives

de l'imaginaire occidental, Montréal, Les Presses de

l’Université de Montréal, 2002, 431 p.

\section{Maurice Boutin}

Volume 19, numéro 2, printemps 2007

Penser sa mort?

URI : https://id.erudit.org/iderudit/017508ar

DOI : https://doi.org/10.7202/017508ar

Aller au sommaire du numéro

Éditeur(s)

Université du Québec à Montréal

ISSN

1180-3479 (imprimé)

1916-0976 (numérique)

Découvrir la revue

Citer ce compte rendu

Boutin, M. (2007). Compte rendu de [Hentsch, Thierry, Raconter et mourir. Aux sources narratives de l'imaginaire occidental, Montréal, Les Presses de

l’Université de Montréal, 2002, 431 p.] Frontières, 19(2), 79-79.

https://doi.org/10.7202/017508ar

Ce document est protégé par la loi sur le droit d'auteur. L'utilisation des services d'Érudit (y compris la reproduction) est assujettie à sa politique d'utilisation que vous pouvez consulter en ligne.

https://apropos.erudit.org/fr/usagers/politique-dutilisation/
Cet article est diffusé et préservé par Érudit.

Érudit est un consortium interuniversitaire sans but lucratif composé de l’Université de Montréal, l'Université Laval et l'Université du Québec à Montréal. Il a pour mission la promotion et la valorisation de la recherche. https://www.erudit.org/fr/ 
sommes poursuivis toute une vie par ce que nous n'avons pas osé vivre en entièreté » (p. 56); "Au cœur d'une maladie mortelle, on peut tout entreprendre avec le temps imparti » (p. 101); "Chaque jour se doit d'être une création nouvelle» (p. 114)

Tout en étant d'une lucidité, d'une intensité de vivre qui font mal, ce livre nous aide à vivre. Des romans et essais d'elle, il faut lire au moins, si ce n'est déjà fait, ses incontournables: La mort viennoise, Une passion, Les sept nuits de la reine, Seul ce qui brûle. C. Singer est décédée à Vienne, le 4 avril 2007. Mais elle vivra tant que nous la lirons. Lisons-la, encore et encore, et offrons ses livres aux jeunes qui se croient immortels.

Hans-Jürgen Greif

\section{HENTSCH, Thierry}

\section{Raconter et mourir}

\section{Aux sources narratives de l'imaginaire occidental}

Montréal, Les Presses de I'Université de Montréal, 2002, $431 \mathrm{p}$.

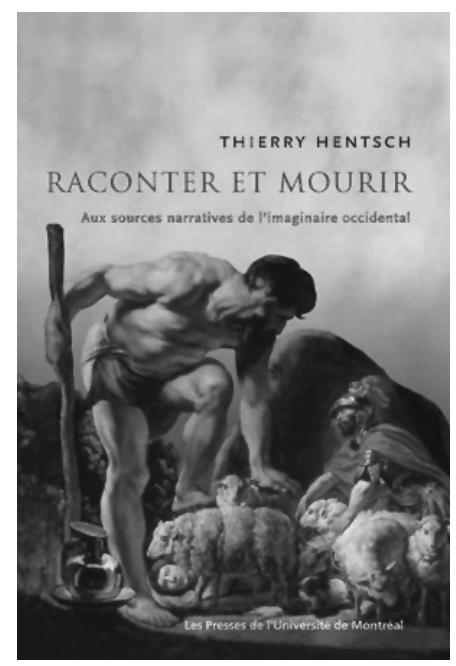

Dans l'introduction, l'auteur annonce ses couleurs en adoptant une posture qui démontre à l'envi tout au long du livre la synthèse impossible à laquelle il choisit de se soumettre et qu'il appelle, de façon pompeuse, "l'imaginaire occidental »: il s'agit de tourner le dos, résolument, à l'avenir. Dans les dernières décennies surtout, l'avenir n'a servi qu'à alimenter des rêves qui ont fait crûment déchanter, et il a abusé ainsi de ce dont l'imaginaire occidental est capable; ou plutôt, mais ce n'est guère mieux, l'avenir attendu a effectivement accompli le destin de cet imaginaire précisément en tant qu'occidental, c'est-à-dire brillant du côté du soleil couchant et distillant donc un parfum de fin de partie, en se donnant, "dans une sorte d'intuition géniale", avec le nom du « couchant » la mort comme horizon (p. 13). Une compréhension à la fois moins superficielle et plus critique de la signification même du mot "Occident » est présentée par Peter Sloterdijk dans Le palais de cristal. À l'intérieur du capitalisme planétaire (Paris, Maren Sell Éditeurs, 2006; original allemand 2005). Ce livre reprend, sous une forme modifiée, la partie conclusive de Sphères II, Globes, Macrosphérologie, un livre publié en 1999 et dont la traduction française prochaine est annoncée. Le vaste projet des Sphères - Sphères I, Bulles, Microsphérologie (1998, traduction française 2002), Globes, et Sphères III, Écumes, Sphérologie plurielle (2004, traduction française 2005), trois volumes qui totalisent plus de 2500 pages - est une tentative de configurer le narratif et le philosophique I'un avec I'autre pour faire en sorte qu'il s'agisse de plus et d'autre chose que de la simple addition de deux visions de borgne, et de fournir, avec les moyens d'un grand récit d'inspiration philosophique, les contours d'une théorie du temps présent d'une manière autrement plus fouillée et complète que ce qui est offert dans Raconter et mourir. La façon dont Sloterdijk réclame et défend la nécessité des "grands récits" encore aujourd'hui est autrement plus convaincante que les accents éplorés de Hentsch sur leur absence actuelle et l'injonction de se défaire des emprises d'un futur qui tourne si vite à l'obsession. Mais ce livre pour bourlingueur fatigué ne fait que proposer un changement d'obsession: il entend procurer un bain d'étrangeté à même le familier devenu insipide à force d'être évident.

Les rubriques suivantes servent de titres aux cinq parties du livre: l. L'immortalité et la vie (p. 35-90); 2. L'épreuve de la connaissance (p. 91-196); 3. La vérité ou la mort (p. 197-259); 4. Héroïsme et vérité (p. 261-326); 5. L'irruption du doute (p. 327-415). Il est question d'Ulysse, d'Énée, et de Gilgamesh (partie I); du récit biblique de la Genèse, d'Hésiode, d'CEdipe-roi, d'Antigone et du Banquet de Platon (partie 2); de I'Évangile et des Confessions d'Augustin (partie 3); de la Chanson de Roland, de Perceval, de Lancelot, de Tristan et de la Divine Comédie de Dante (partie 4); de Rabelais, du Don Quichotte de Cervantès, de Hamlet et de Descartes (partie 5).

Si l'auteur achoppe sur le paradoxe qu'il nomme "récit de la vérité » qui ne vit que du dualisme oppositionnel temps (= récit)/éternité (= vérité), allant même jusqu'à en faire l'énigme centrale, selon lui à peine avouable, du christianisme, c'est qu'il oublie que « récit et vérité » n'est pas l'équivalent de " raconter et mourir ». Pareil rapprochement signale - et rappelle aussi - que la narration est travaillée de I'intérieur par ce qui la dépasse, par quelque chose d'inassimilable qui lui confère pourtant un ordonnancement qui vaut comme sens.

La préférence de l'auteur pour la thématique fait qu'il néglige l'importance de l'écriture, même s'il en parle souvent. Mais ces nombreux développements tournent facilement à la surenchère et ne parviennent pas à faire ombrage à la thématique du "et» dans le titre choisi: Raconter et mourir. Si cet «et" n'est d'aucun temps, ce n'est pas par l'effet d'un coefficient d'éternité qui tournerait le dos à I'histoire, mais c'est bien plutôt qu'il est de tous les temps et qu'il se retrouve donc, du moins dans l'imaginaire occidental qui est I'unique souci de l'auteur, dans les récits qu'il choisit d'analyser et qui montrent à quel point, dans cet ailleurs à l'occidentale, la source ne bruit que dans les marges. Seraitce donc que la mise en récit d'une vérité fait d'emblée de celle-ci une chimère et signale l'échec toujours annoncé d'une identité qui tenterait en vain de s'arracher à l'éphémère de la vie?

L'auteur tente d'endiguer son ambitieux projet - rien moins qu'un retour aux sources de l'imaginaire occidental - en s'arrêtant à Descartes chez qui sa lecture " trouve matière ou prétexte à conclure » (p. 397) ce pèlerinage aux sources. Tout ce qui vient après Descartes ne peut être que "faille mortelle dans la vérité » que Descartes entendait pourtant "sauver» (p. 415). Après Descartes, il n'y aurait place que pour des "ambitions démesurées et des espoirs destructeurs "; il est donc plus que temps, selon l'auteur, de tirer enfin les conclusions qui s'imposent quant à la croyance au progrès qui "s'étiole" sous nos yeux aujourd'hui, et de mieux discerner «ce qu'il en coûte d'avoir cru pouvoir rapatrier en ce monde la promesse de salut et la vérité que le christianisme réservait pour l'au-delà de la mort» (p. 415). C'est sur ce vœu que se clôt cette "série d'essais sur l'imaginaire occidental » (p. 4) qui témoigne une fois de plus - une fois de trop! - que nous vivons bien dans une culture qui ne peut pratiquement pas parler du plus manifeste si ce n'est sous la forme de la distinction grossière entre la bonne et la mauvaise ambiance.

La question qui demeure tout au long de la lecture de ce livre est celle-ci: I'auteur a-t-il vraiment quelque chose de neuf à dire, ou s'est-il plutôt contenté de citer une multitude d'auteurs dans le but d'égayer quelque peu un propos franchement répétitif qui se complaît et s'épuise à dénoncer un imaginaire « dominé par la peur du manque, la hantise de la maladie et le refus de la mort " (p. 421)? Ce livre est en tout cas une forte incitation à changer sa liste de lectures.

Raconter et mourir - c'est-à-dire, selon le "post-scriptum» du livre achevé d'imprimer en septembre 2002, "mourir apaisé d'avoir vécu et transmis» (p. 420). C'est, malgré tout, ce qu'il y a encore de mieux à espérer pour l'Auteur qui devait décéder moins de trois ans plus tard, le 7 juillet 2005.

Maurice Boutin

\section{BAUD, René-Claude \\ Ce qui remonte de l'ombre} Itinéraire d'un soignant

Paris, Bayard, 2006, 163 p.

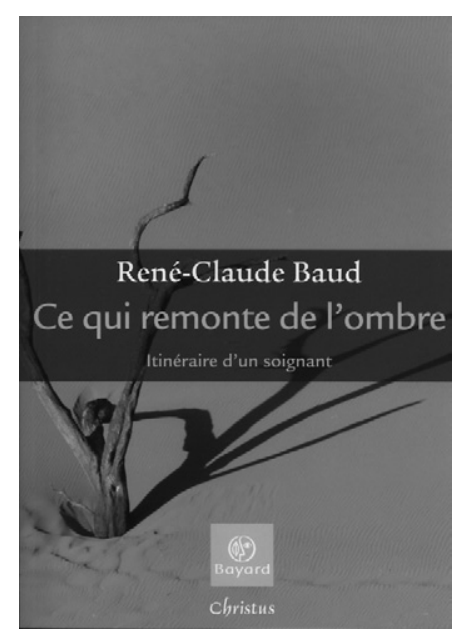

Jésuite (p. 11), diplômé en philosophie et en théologie (p. 63), ancien aumônier dans un collège pour adolescents (p. 62 et 153), aide-soignant de nuit, pendant vingt ans (p. 46), auprès de malades atteints du sida (p. 10), responsable de la formation d'accompagnants en soins palliatifs ( $p .126$ ) et fondateur de l'association Albatros à Lyon (quatrième de couverture), 